\title{
Diagnóstico de bacias hidrográficas para a sustentabilidade - diagnóstico rápido participativo como estratégia de mobilização para a gestão integrada e participativa no Estado do Rio de Janeiro, com ênfase na região da Foz do Rio Paraíba do Sul
}

\author{
Maria Inês Paes Ferreira ${ }^{1}$ \\ José Augusto Ferreira da Silva ${ }^{1}$ \\ Ricardo Pacheco Terra ${ }^{2}$ \\ Karla Aguiar Kury ${ }^{2}$ \\ Thiago Caetano da Silva Berriel ${ }^{2}$ \\ Maria Eugênia Totti ${ }^{3}$ \\ Mariana Rodrigues de Carvalhaes Pinheiro ${ }^{1}$ \\ ${ }^{1}$ Instituto Federal Fluminense - IFF \\ Rodovia Amaral Peixoto, km 164, Imboacica, - Macaé - RJ, Brasil. \\ ines_paes@yahoo.com.br \\ ${ }^{2}$ Instituto Federal Fluminense - IFF \\ Rua Doutor Pereira de Siqueira, 273 - Parque Dom Bosco - Campos dos Goytacazes, RJ. \\ Brasil - CEP 28030-130. \\ riterra@gmail.com; \\ ${ }^{3}$ Universidade Estadual Norte Fluminense - UENF \\ Endereço - Campos dos Goytacazes - RJ, Brasil. \\ meftotti@gmail.com

\begin{abstract}
The objective of this article is to relate the final results of several workshops conducted during 2008, which integrated the Course for social representations in the northern region of Rio de Janeiro State in hydrographic basins management. Rapid Participative Diagnosis was employed to evaluate environmental, social technical and political problems conflicts and potentialities related to the management of water resources in the Paraíba do Sul Basin estuary. The results, obtained during workshops realized with Basin Committee members of the Ninth Hydrographic Region of Rio de Janeiro State, Brasil (RH IX) pointed out the need for improving interinstitutional transescalars connections among Paraíba do Sul Basin's Federal Committee (CEIVAP) and State Committee (Low Paraíba do Sul River Committee), as well as stimulating collective consensus on reference conditions for the elaboration a The Basin Director Plan which contemplates Rio de Janeiro's representations' needs. It was also depicted that the valuing of environmental services, as well as implementation of integrated hydric resources monitoring systems can be regarded as important strategies for guiding Public Policies and improving environmental quality, not only for the RH IX, but also for all Rio de Janeiro's Hydrographic Regions. Flood control related emergency measures were picked out as priority.
\end{abstract}

Palavras-chave: Região Hidrográfica IX do Estado do Rio de Janeiro; Bacia do Rio Paraíba do Sul; Diagnóstico Rápido Participativo: Problemas, Conflitos e Potencialidades; Valoração de Serviços Ambientais.

Key words: Ninth Hydrographic Region of Rio de Janeiro State; Paraíba do Sul Basin; Participative Rapid Diagnostic; Problems, Conflicts and Potentialities; Environmental services valuation.

\section{Introdução}

Os problemas e conflitos em torno do uso dos recursos hídricos apresentam-se cada vez mais complexos, ampliando as disputas, tanto entre bacias hidrográficas quanto dentro de uma mesma bacia. Conflitos socioambientais podem ser entendidos como disputas entre grupos sociais derivadas dos distintos tipos de relações por eles mantidas com seu meio natural. Há três dimensões básicas a serem consideradas no entendimento e na análise desses conflitos: o mundo biofísico e os ciclos naturais, o mundo humano e suas estruturas sociais e o relacionamento dinâmico, interdependente, entre estes dois mundos. Ocorrem conflitos pelo 
controle dos recursos naturais, conflitos derivados dos impactos ambientais e sociais decorrentes de determinados usos, e também aqueles ligados aos usos e apropriações dos conhecimentos ambientais (LITTLE, 2001).

Nesse sentido, o presente trabalho tem como objetivo identificar e classificar problemas e conflitos encontrados no processo de Gestão dos Recursos Hídricos no Estado do Rio de Janeiro, e também propor alternativas para a sua superação, utilizando o Diagnóstico Rápido Participativo (DRP) como ferramenta. O trabalho sistematiza os resultados das atividades práticas e das Oficinas de DRP realizadas no ano de 2008 com as representações do Comitê de Bacia do Baixo Paraíba do Sul (CBBPS) e membros da sociedade civil, pertencentes principalmente a Organizações Não-governamentais (ONGs) e ao Poder Público local da Região Hidrográfica IX do Estado do Rio de Janeiro. Essas atividades integraram o Curso "Capacitação e mobilização de representações do norte-noroeste fluminense para criação de Organismos de Bacia na Região Hidrográfica IX do Estado do Rio de Janeiro", executado pelo IF Fluminense, com apoio do CNPq, do MCT-CTHidro e da ANA (Edital 30/07), com vistas a atender à demanda de capacitação regional de profissionais atuando em instituições públicas, em Organizações Não Governamentais (ONGs) e demais representações sociais envolvidas com a gestão de recursos hídricos do norte-noroeste fluminense, de forma a promover a participação qualificada das representações no Organismo de Bacia na Região Hidrográfica IX (RH IX) do Estado do Rio de Janeiro, bem como a estimular e fornecer subsídios técnicos para a atuação da sociedade junto aos órgãos responsáveis pelo gerenciamento dos recursos hídricos. Os pressupostos metodológicos do curso se basearam nas diretrizes do IBAMA para projetos de educação no processo de gestão ambiental (QUINTAS, 2000) e na construção do referencial teórico para apoiar os módulos temáticos do curso. O material didático produzido culminou na publicação do quarto Boletim do Observatório Ambiental Alberto Ribeiro Lamego (2008b).

\section{Metodologia}

A metodologia adotada constitui-se numa adaptação do método Zoop (Planejamento de Projetos Orientado por Objetivos) ${ }^{1}$, para facilitar a obtenção de informações rápidas e por escrito. O Diagnóstico Rápido Participativo $(\mathrm{DRP})^{2}$ levantou problemas, conflitos e potencialidades relativas à gestão de recursos hídricos no Estado do Rio de Janeiro (Grupo 1) e na Região Hidrográfica IX (Grupo 2). Cada um dos grupos procedeu à classificação e à hierarquização de problemas/conflitos ${ }^{3}$. Para classificação e hierarquização dos problemas/conflitos de naturezas técnica, social, política e/ou ambiental, registrados individualmente para cada participante da Oficina de DRP, considerou-se a frequência $(F)$ do problema, a sua gravidade $(\mathrm{G})$ e a factibilidade de solução (S) do mesmo, perante os conhecimentos tecnológicos disponíveis na região, de acordo com os critérios apresentados no quadro 1.

\footnotetext{
${ }^{1}$ Metodologia criada entre as décadas de 70 e 80 pela Agência Alemã de Cooperação Técnica (GTZ), visando privilegiar a participação social, através do trabalho em grupo, nos processos de planejamento e gestão de projetos voltados ao desenvolvimento (SALLES e ROSA, 2008).

${ }_{2}^{2}$ DRP é um processo de apreensão da visão da sociedade sobre a realidade, com base em um conjunto de ferramentas, para conhecer, avaliar e planejar idéias, problemas, oportunidades, obstáculos locais e desenvolvimento regional, gerando resultados rápidos...(TAKARA, 2007).

${ }^{3}$ Um problema é caracterizado por uma situação onde não há nenhuma reação por parte dos atingidos; conflito é caracterizado pelo confronto de interesses em relação à utilização ou gestão de um recurso (QUINTAS, 2000).
} 


\begin{tabular}{|l|l|l|l|}
\hline Item & Grau 1 & Grau 2 & Grau 3 \\
\hline Frequência (F) & Baixa & Média & Alta \\
\hline Gravidade (G) & Pouca & Moderada & Acentuada \\
\hline Solução (S) & Fácil & Moderada & Difícil \\
\hline
\end{tabular}

Quadro 1. Hierarquização de problemas e conflitos.

Tendo em vista a diversidade de representações da RH-IX, o curso foi concebido em estrutura modular, e ministrado em quatro municípios, das quatro principais sub-bacias da região, doravante denominados "Núcleos": o Núcleo Quissamã (para as representações da bacia da Lagoa Feia/Macabu/Ururaí-Imbé); Núcleo Campos dos Goytacazes I (para as representações da Foz do Rio Paraíba do Sul e dos canais da baixada campista); Núcleo Campos dos Goytacazes II (para as representações da bacia dos Rios Muriaé, Pomba e adjacentes) e o Núcleo Macaé (para representações de outras Regiões Hidrográficas do Estado do Rio e Janeiro). O curso totalizou oito momentos presenciais de $8 \mathrm{~h}$ cada, distribuídas ao longo de 1 ano, em módulos semanais alternados por Núcleo, com exceção do último módulo, que contou com a participação das representações dos quatro Núcleos em uma única oficina para a sistematização dos resultados do DRP.

\section{Resultados e Discussão}

No âmbito estadual, foram identificados os seguintes fatores que comprometem a quantidade e a qualidade dos mananciais: (i) falta de controle da ocupação urbana nas suas proximidades; (ii) aumento do volume de esgotamento sanitário não tratado e aumento na demanda por redes de abastecimento de água; (iii) falta de cobertura vegetal e consequentemente erosão e assoreamento dos rios, bem como o desmatamento e desflorestamento de galerias e ciliares; (iv) outras fontes de degradação da bacia, como poluição difusa, falta de controle adequado dos poluentes orgânicos (atividades rurais), poluição doméstica e poluição industrial; (v) conflitos devidos ao interesse de empresas na produção e comercialização de água potável e na distribuição de energia elétrica; (vi) conflitos político-administrativos entre vários níveis da administração Municipal, Estadual e Federal, bem como entre organizações partidárias; (vii) conflitos entre representações das próprias bacia, no que diz respeito à disponibilidade, à qualidade, à cobrança e aos diferentes tipos de uso e interesses; e (viii) falta de conhecimento por parte das populações direta ou indiretamente envolvidas nos processos de gestão dos recursos hídricos e outras questões ambientais. Os problemas e conflitos são, em sua grande maioria, de origem social ou econômica, mas também, podem estar atrelados a questões de outra natureza, como a questão política, a ambiental ou a própria questão técnica; muitas vezes fica difícil defini-los como pertencentes a um único fator, pois apresentam envolvimento de várias dimensões. A tabela 1 apresenta os resultados dos problemas e conflitos relativos à gestão dos recursos hídricos no âmbito estadual levantados no DRP produzido pelo Grupo 1. 
Tabela 1. Problemas e conflitos relativos à gestão dos recursos hídricos no Estado do Rio de Janeiro, levantados no DRP produzido pelo Grupo 1.

\begin{tabular}{|c|c|c|c|c|c|c|c|c|c|c|}
\hline \multirow{3}{*}{ 苞 } & \multicolumn{5}{|c|}{ Problemas } & \multicolumn{5}{|c|}{ Conflitos } \\
\hline & \multirow[b]{2}{*}{ Descrição } & \multicolumn{4}{|c|}{ Hierarquização } & \multirow{2}{*}{ Descrição } & \multicolumn{4}{|c|}{ Hierarquização } \\
\hline & & $\mathrm{F}$ & G & $\mathrm{S}$ & Total & & $\mathrm{F}$ & G & $\mathrm{S}$ & Total \\
\hline \multirow{4}{*}{ 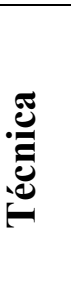 } & $\begin{array}{l}\text { Tratamento de } \\
\text { esgoto/distribuição da água }\end{array}$ & 3 & 3 & 2 & 8 & & & & & \\
\hline & Assoreamento e erosão & 2 & 2 & 2 & 6 & & & & & \\
\hline & $\begin{array}{l}\text { Poluição difusa /poluição } \\
\text { orgânica (atividades rurais) }\end{array}$ & 3 & 3 & 1 & 7 & & & & & \\
\hline & Poluição industrial & 3 & 3 & 2 & 8 & & & & & \\
\hline \multirow{3}{*}{ 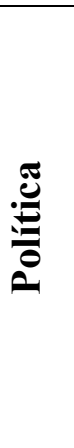 } & & & & & & $\begin{array}{l}\text { Interesses pela produção } \\
\text { e/ou distribuição de água } \\
\text { potável* }\end{array}$ & 2 & 2 & 2 & 6 \\
\hline & & & & & & $\begin{array}{l}\text { Conflitos entre órgãos } \\
\text { administrativos } \\
\text { municipais, estaduais e } \\
\text { federais* }\end{array}$ & 3 & 3 & 3 & 9 \\
\hline & & & & & & $\begin{array}{l}\text { Conflitos de interesses } \\
\text { entre os representantes } \\
\text { das bacias que compõem } \\
\text { as RHs* }\end{array}$ & 3 & 2 & 2 & 7 \\
\hline 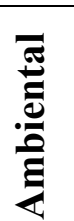 & & & & & & $\begin{array}{l}\text { Disputas pela produção } \\
\text { de energia elétrica*** }\end{array}$ & 3 & 3 & 3 & 9 \\
\hline 吾 & $\begin{array}{l}\text { Desconhecimento de } \\
\text { informação sobre questões } \\
\text { ambientais }\end{array}$ & 3 & 3 & 1 & 7 & & & & & \\
\hline
\end{tabular}

Notas: * - com interações na dimensão social; ** - com interações nas dimensões política e social.

Legenda: F- frequência; $\mathrm{G}$ - gravidade; $\mathrm{S}$ - solução

O Grupo 2 sistematizou os resultados da Oficina de DRP, com foco na RH IX, conforme apresentado na tabela 2. Os núcleos participantes diagnosticaram alguns problemas e conflitos semelhantes apesar de apresentarem visões divergentes sobre eles. Dentre os problemas e conflitos levantados, destaca-se a manutenção dos canais da baixada campista, que envolveu dimensões técnicas, políticas, ambientais e sociais. A manutenção dos canais inclui as ações de manejo das comportas, a definição dos trechos sujeitos a dragagem, a destruição e restauração de diques, entre outras intervenções. Contudo, os diagnósticos divergem quanto ao modo de intervenção e aos setores beneficiados, evidenciando a gravidade do conflito e a dificuldade em solucioná-lo. Diante das demais constatações do grupo, infere-se que o conflito dos canais é consequência da ausência de diálogo e enfrentamento dos setores envolvidos, uma vez que divergências quanto à forma do uso do solo e dos recursos hídricos foram discutidas de forma incipiente até o momento. Portanto, torna-se indispensável o fortalecimento de um fórum participativo que considere todas as dimensões envolvidas no problema/conflito, as diretrizes nacionais e estaduais para atuação na solução do 
problema/conflito e a garantia da participação equilibrada dos atores que representam os múltiplos interesses envolvidos nos problemas e nos conflitos.

Tabela 2. Hierarquização dos problemas ou conflitos gerais da RH-IX do Estado do Rio de Janeiro.

\begin{tabular}{|c|c|c|c|c|c|c|c|c|c|c|}
\hline \multirow{3}{*}{ 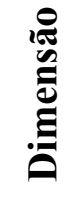 } & \multicolumn{5}{|c|}{ Problemas } & \multicolumn{5}{|c|}{ Conflitos } \\
\hline & \multirow[t]{2}{*}{ Descrição } & \multicolumn{4}{|c|}{ Hierarquização } & \multirow[t]{2}{*}{ Descrição } & \multicolumn{4}{|c|}{ Hierarquização } \\
\hline & & $\mathrm{F}$ & $\mathrm{G}$ & $\mathrm{S}$ & Total & & $\mathrm{F}$ & $\mathrm{G}$ & $\mathrm{S}$ & Total \\
\hline \multirow{3}{*}{ •ֶֶ } & $\begin{array}{l}\text { Manutenção dos canais da } \\
\text { baixada campista (canais e } \\
\text { diques) }\end{array}$ & 3 & 3 & 3 & 9 & $\begin{array}{l}\text { Definição dos canais e } \\
\text { respectivos trechos a serem } \\
\text { dragados }\end{array}$ & 2 & 3 & 3 & 8 \\
\hline & $\begin{array}{l}\text { Ameaças aos grandes } \\
\text { mananciais e fluxos de água } \\
\text { doce* }\end{array}$ & 3 & 3 & 3 & 9 & 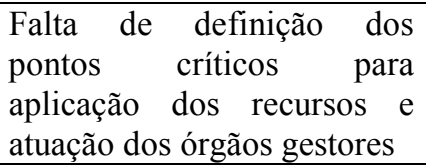 & 2 & 1 & 1 & 4 \\
\hline & Ameaças à costa marítima* & 2 & 2 & 2 & 6 & & & & & \\
\hline \multirow{4}{*}{ 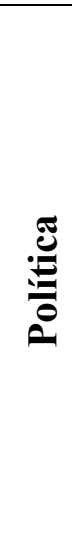 } & $\begin{array}{l}\text { Manejo das comportas da } \\
\text { baixada campista }\end{array}$ & 2 & 3 & 3 & 7 & $\begin{array}{l}\text { Abertura e fechamento das } \\
\text { comportas }\end{array}$ & 2 & 3 & 3 & 7 \\
\hline & $\begin{array}{l}\text { Falta de Câmaras técnicas } \\
\text { trans e/ou multidisciplinares } \\
\text { no OB }\end{array}$ & 3 & 3 & 3 & 9 & $\begin{array}{l}\text { Falta de consenso quanto aos } \\
\text { objetivos de gestão a serem } \\
\text { atingidos na RH IX }\end{array}$ & 2 & 2 & 2 & 6 \\
\hline & \multirow[t]{2}{*}{$\begin{array}{l}\text { Descontinuidade nas ações } \\
\text { nas mudanças de governo e } \\
\text { aplicação dos recursos } \\
\text { somente nas emergências }\end{array}$} & 3 & 3 & 3 & 9 & $\begin{array}{l}\text { Divergências quanto à forma } \\
\text { do uso e ocupação dos solos } \\
\text { e dos recursos hídricos }\end{array}$ & 3 & 3 & 2 & 8 \\
\hline & & & & & & $\begin{array}{l}\text { Ações governamentais } \\
\text { falhas, resultando na } \\
\text { insatisfação dos diversos } \\
\text { setores e em descrédito }\end{array}$ & 3 & 2 & 3 & 8 \\
\hline \multirow{2}{*}{ 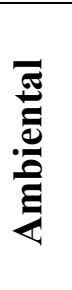 } & Inundação & 2 & 2 & 3 & 7 & \multirow{2}{*}{$\begin{array}{l}\text { Os atores } \\
\text { raramente tenvolvidos } \\
\text { legítimos, interesses } \\
\text { temática ambiental e os } \\
\text { espaços para promoção } \\
\text { política }\end{array}$} & & & & \\
\hline & $\begin{array}{l}\text { Falta de equilíbrio entre a } \\
\text { necessidade de se preservar e } \\
\text { de produzir o alimento }\end{array}$ & 2 & 2 & 1 & 5 & & & & & \\
\hline \multirow[b]{2}{*}{ 矛 } & $\begin{array}{l}\text { Ocupação irregular } \mathrm{de} \\
\text { FMPs* }\end{array}$ & 3 & 3 & 3 & 9 & $\begin{array}{l}\text { Derrubada dos diques } \\
\text { irregulares }\end{array}$ & 1 & 3 & 3 & 7 \\
\hline & $\begin{array}{l}\text { Falta de geração de renda } \\
\text { própria levando ao subsídio } \\
\text { permanente dos programas } \\
\text { sociais do governo }\end{array}$ & 3 & 3 & 1 & 7 & $\begin{array}{l}\text { Interferência nas decisões } \\
\text { por parte de ambientalistas } \\
\text { radicais, desconsiderando os } \\
\text { demais atores envolvidos }\end{array}$ & 2 & 2 & 2 & 6 \\
\hline
\end{tabular}

Legenda: F- freqüência; $\mathrm{G}$ - gravidade; $\mathrm{S}$ - solução.

Notas: * - com interações na dimensão ambiental; FMPs - faixas marginais de proteção. 
Tendo em vista os problemas e conflitos apontados tanto para a gestão das águas no Estado do Rio de Janeiro como para sua RH IX, é necessário pensar em estratégias para a integração dos atores sociais do Comitê do Baixo Paraíba do Sul às representações do Comitê de Integração da Bacia Hidrográfica do Rio Paraíba do Sul (CEIVAP). Nesse sentido, compatibilizar as prioridades de um Plano de Bacia coletivamente construído com foco local com o Plano Diretor de Recursos Hídricos da Bacia do Rio Paraíba do Sul é prioridade para o processo de implantação do Organismo de Bacia $(\mathrm{OB})$ estadual, ora em curso. A superação desse desafio depende do respeito às bases conceituais da Política Nacional de Recursos Hídricos (PNHR), que preconiza a paridade e a participação qualificada dos três setores que compõem um OB (BRASIL, 1997). A bacia hidrográfica é a unidade natural que permite integração institucional e articulação da pesquisa com o gerenciamento, e possibilita ainda implantar um banco de dados que funcionará como uma plataforma para o desenvolvimento de projetos com alternativas, levando-se em conta os custos destas. Para que tal potencialidade seja adequadamente desenvolvida na Bacia do Paraíba do Sul, a efetividade das representações regionais da RH IX no CEIVAP deve ser garantida. Destaca-se que, no Setor Sociedade Civil, há apenas uma representação do Comitê da Foz do Paraíba do Sul com assento, indicando a necessidade de comunicação e de articulação constantes entre os diversos atores do CEIVAP para que as demandas da região da Foz do Paraíba, atingida por contaminação e acidentes ambientais diversos nos últimos anos, sejam de fato contempladas. Paralelamente, a capacitação de gestores deve avançar, no sentido do gerenciamento integrado.

Com relação aos canais da baixada campista, os participantes do DRP destacaram que, além dos problemas de governança relacionados às práticas centralizadoras de gestão, implementadas anteriormente na região pelos órgãos gestores, na inexistência do OB de nível estadual (criado pelo Poder Público em novembro de 2008), os problemas e os conflitos de gerenciamento solucionados com o emprego de estratégias concebidas de forma fragmentada e em níveis local e/ou setorial causaram impactos negativos para as comunidades da Foz do Rio Paraíba do Sul. A percepção dos participantes é de que tais impactos só serão adequadamente mitigados por meio de ações associadas a um modelo de gerenciamento integrado, com visão ecossistêmica e não meramente "hidráulica", e que estimule o estabelecimento de conexões institucionais transescalares (VIEIRA et al., 2005) envolvendo o Poder Público, os usuários e a Sociedade Civil. A participação dos usuários, do público e da iniciativa privada, que deve ser um dos eixos principais da governança dos recursos hídricos, complexifica-se no contexto da RH-IX do Estado do Rio de Janeiro, constituída por bacias de dominialidade estadual, como a da Lagoa Feia (com as sub-bacias dos Rios Macabu e do Imbé/Ururaí), e pela foz de uma bacia de dominialidade federal de extrema relevância para o país - a Bacia do Rio Paraíba do Sul, que possui especificidades nas suas margens esquerda e direita, associadas aos sistemas de canais artificiais. Minimizar a assimetria na qualidade e quantidade de representações, em situação de elevada heterogeneidade, tanto com relação à RH-IX como à Bacia do Paraíba, implica intensificar as ações da Agência Nacional de Águas no Estado do Rio de Janeiro, apoiando as ações estaduais na elaboração de projetos que visem à recuperação e à proteção dos recursos hídricos da Foz do Paraíba, cujas comunidades vêm sendo constantemente impactadas por fatores climáticos associados ao gerenciamento inadequado dos canais. As discussões desses episódios podem ser consultadas nos Boletins do Observatório Ambiental Alberto Ribeiro Lamego referentes aos anais do I Seminário Regional sobre Gestão de Recursos Hídricos nas Regiões Hidrográficas das Bacias dos rios Macaé, das Ostras, lagoas costeiras do Norte Fluminense e da foz do rio Paraíba do Sul (FERREIRA et al., 2008a) e ao eixo temático "a qualidade ambiental associada à gestão dos recursos hídricos na Região Hidrográfica IX do 
Estado do Rio de Janeiro" (FERREIRA et al., 2008b). Cabe mencionar que, dada a importância nacional da Bacia do Rio Paraíba do Sul, as regiões hidrográficas a ela relacionadas devem ser contempladas de maneira especial no Plano Estadual de Recursos Hídricos (PEHR), cujo Termo de Referência encontra-se em fase de consolidação. A proposta do Poder Público Estadual é elaborar um PERH contemplando estratégias de gestão da água, a curto, médio e longo prazos, construído de forma participativa e que aponte diretrizes para a gestão integrada das bacias hidrográficas. Assim, urge incrementar a atuação de Câmaras Técnicas nos Organismos de Bacia Estaduais, e sua interação com aquelas já em funcionamento em Organismos de Bacia de dominialidade federal (como o do Rio Paraíba do Sul, cuja gestão integrada é competência última do CEIVAP). Ressalta-se que o processo de implementação do Comitê do Baixo Paraíba do Sul está em consonância com essa percepção. Incrementar a capacidade de monitoramento avançado dos recursos hídricos, com vistas à elaboração de bancos de dados integrados e à produção de ferramentas de suporte à decisão adequados à gestão é outra estratégia a ser incentivada. Na RH IX também é importante detectar fragmentos florestais e presença de vegetação ciliar associados a propriedades particulares ou comunais que possam ser enquadrados em programas de compensação às populações pelos serviços ambientais de conservação/proteção de nascentes e corpos hídricos (do tipo "produtores de água", vistos pelas representações de agricultores como uma boa solução para os conflitos relacionados à ocupação irregular de faixas marginais de proteção - FMPs). Paralelamente, está em andamento um projeto de valoração dos serviços ambientais do Domínio de Ilhas Fluviais do Paraíba do Sul (DIFs), como subsídio ao estabelecimento de Políticas Públicas para indenização dos pescadores artesanais afetados por pequenas centrais hidrelétricas em fase de licenciamento na Bacia. Para realização desses estudos de valoração, a proposta metodológica sugerida tem como objetivos: (i) gerar informação técnico-científica para evidenciar a importância dos DIFs para subsidiar ações para sua proteção; (ii) sistematizar o debate acerca do conjunto de impactos gerados a partir da implantação de UHEs na região dos DIFs; (iii) investigar o potencial de conexões institucionais transescalares (via Diagrama de Venn); (iv) propor metodologia para valoração ambiental dos impactos gerados com a implantação de empreendimentos hidrelétricos na região dos DIFs; e (v) iniciar os estudos de ressarcimento às populações tradicionais "produtoras de água".

Por outro lado, mesmo reconhecendo que o diálogo de saberes entre atores sociais diversos é vital para garantir a sustentabilidade dos recursos hídricos, alguns participantes da oficina enfatizaram que novas tecnologias, com soluções que incluem os usos de sistemas de processos naturais, como a ecotecnologia e a ecohidrologia, devem ser também investigadas, com vistas à aplicação na região.

\section{Conclusões}

A sistematização dos resultados das atividades realizadas junto às representações da RH IX e aos demais participantes do curso de capacitação e das atividades de mobilização coordenadas pelas Instituições de Ensino e Pesquisa participantes do CBH do Baixo Paraíba do Sul indica a necessidade de implementar abordagens preditivas de gerenciamento, pesquisa e elaboração de banco de dados baseadas em programas densos e tecnicamente avançados de monitoramento, abordagens essas apoiadas num sistema adequado de governança de água com a finalidade de promover oportunidades de desenvolvimento regional e sustentável a partir do estabelecimento de cenários de disponibilidade e demanda hídricas. Tais abordagens devem também contemplar a valoração dos "serviços" dos ecossistemas aquáticos e dos recursos hídricos para proposição de compensação aos "produtores de água", estratégia ímpar de mobilização de segmentos populares para 
participar na gestão integrada das águas, solucionar problemas de ocupação irregular de FMP e contemplar, no caso da RH-IX, ações de articulação e de fortalecimento de conexões interinstitucionais transescalares entre o CEIVAP e o Comitê do Baixo Paraíba do Sul. Espera-se assim caminhar na direção da revisão do Plano de Bacia, elaborado em condições de inexistência do OB estadual, e da participação no processo de construção do Plano Estadual de Recursos Hídricos.

\section{Referências}

BRASIL. Lei federal n. 9.433 de 8 de janeiro de 1997 que institui a política nacional de recursos hídricos. Brasília. Legislação Ambiental/MMA, 1997.

BRASIL. Lei $\mathbf{n}^{\circ}$ 9.433, de 8 de Janeiro de 1997. Institui a Política Nacional de Recursos Hídricos, cria o Sistema Nacional de Gerenciamento de Recursos Hídricos, regulamenta o inciso XIX do art. 21 da Constituição Federal e altera o art. $1^{\circ}$ da Lei 8.001, de 12 de Março de 1990, que modificou a Lei ${ }^{\circ} 7.990$ de 28 de dezembro de 1989.Brasília, 1997.

FERREIRA, M.I.P., Silva, Pinheiro, M.R.C., Souza, T.C. (Orgs). Anais do I Seminário Regional sobre Gestão de Recursos Hídricos nas Regiões Hidrográficas das Bacias dos rios Macaé, das Ostras, lagoas costeiras do Norte Fluminense e da foz do rio Paraíba do Sul. . Boletim do Observatório Ambiental Alberto Ribeiro Lamego. Campos dos Goytacazes: Essentia Editora. v. 1, n. 2. p 1-197. 2007.

FERREIRA, M.I.P., Silva, Pinheiro, M.R.C., Souza, T.C. (Orgs). A qualidade ambiental associada à gestão dos recursos hídricos na Região Hidrográfica IX do Estado do Rio de Janeiro. Boletim do Observatório Ambiental Alberto Ribeiro Lamego. Campos dos Goytacazes: Essentia Editora. v. 2, n. 1. p 1-150. 2008 a.

FERREIRA, M.I.P., Silva, J.A.F., LUGON Junior, Jr., RODRIGUES, P.P.G.W., PINHEIRO, M.R.C., KURY, K.A., WERNECK, B.R., RODRIGUES, S.M., LIMA, J.F.G., COSTA, R.P. SIQUEIRA, J.M. (Orgs). Capacitação e mobilização de representações do Norte-Noroeste Fluminense para criação de Organismos de Bacia na Região Hidrográfica IX do Estado do Rio de Janeiro. Boletim do Observatório Ambiental Alberto Ribeiro Lamego. Campos dos Goytacazes: Essentia Editora. v. 2, n. 2. p 1-294. 2008 b.

LITTLE, P. Os conflitos socioambientais: um campo de estudo e ação política. In Bursztin, M. (org.) A difícil sustentabilidade - política energética e conflitos ambientais. Rio de Janeiro: Editora Garamond, 2001.

QUINTAS, José Silva. Pensando E Praticando a Educação Ambiental na Gestão do Meio Ambiente. Brasília: Ed. IBAMA, 2000.

SALES, Mara Telles; ROSA, Maria de Fátima Ocani. Análise Comparativa de Metodologias de Intervenção Social: Contribuições Para os Investimentos Sociais das Empresas. In: IV Congresso Nacional de Excelência em Gestão: Responsabilidade Socioambiental das Organizações Brasileiras. Niterói: 2008.

SALES, Mara Telles; ROSA, Maria de Fátima Ocani. Análise Comparativa de Metodologias de Intervenção Social: Contribuições Para os Investimentos Sociais das Empresas. In: IV Congresso Nacional de Excelência em Gestão: Responsabilidade Socioambiental das Organizações Brasileiras. Niterói: 2008.

TAKARA, Tathiana. Ecoturismo de Base Comunitária: Programa de Monitoramento Participativo aplicado ao Projeto Natur (Natureza Turística de Rosana). Trabalho de Conclusão do Curso de Graduação em Turismo. Rosana: UNESP, 2007.

VIEIRA, P. F., BERKES, F., SEIXAS, C. S. Gestão Integrada e Participativa de Recursos Naturais: Conceitos, Métodos e Experiências. Cap. 9. Florianópolis: APED, 2005. 